\title{
Self-lubricant behaviour of copper-carbon nanocomposites: An electron microscopy and atomic force microscopy study
}

Silva, D. , Jeremias, J. , Nunes, D. , Correia, J.B. ${ }^{* * *}$, Hanada, K. ${ }^{* * * * *}$, Colaço, R. ${ }^{* * * * * *}$ and Carvalho, P.A.*

* ICEMS, Instituto Superior Técnico, Av. Rovisco Pais, 1049-001 Lisboa, Portugal.

** Associação Euratom/IST, Instituto de Plasmas e Fusão Nuclear, Instituto Superior Técnico, Av. Rovisco Pais, 1049-001 Lisboa, Portugal.

${ }^{* * * *}$ LNEG, Estrada do Paço do Lumiar, 1649-038 Lisboa, Portugal.

National Institute of Advanced Industrial Science and Technology, 1-2-1 Namiki, Tsukuba, 305-8564, Japan.

CQE, Departamento de Bioengenharia, Instituto Superior Técnico, Av. Rovisco Pais, 1049-001 Lisboa, Portugal.

Email: pac@ist.utl.pt

A microstructure refined to the nanometer scale improves mechanical strength. Furthermore, in the case of carbon dispersions in metals, possible reinforcing effects can be combined with specific properties of carbon phases, such as self-lubricating properties for nanodiamond and graphite [1,2]. High-energy milling is a powder metallurgy process extensively employed to produce nanostructured materials as well as fine particle dispersions in metallic matrices [3]. Nevertheless, continued milling is known to induce amorphization of graphite [4] and to some extent the same can be expected for other carbon allotropes, altering the intrinsic properties of these phases. Furthermore, combination of carbon phases with metallic matrices at the nanoscale represents a fundamental challenge: some metals, like copper, exhibit an intrinsically difficult bonding with carbon phases, which may result in weak interfacial boundaries. Furthermore, the consolidation techniques used must preserve the nanostructure in the composite materials. Suitable processing windows for the dispersion of nanodiamond $(\mathrm{nD})$ and graphite $(\mathrm{G})$ in nanostructured copper by ball milling have been previously established [5] and consolidation has been achieved by either extrusion spark plasma sintering (SPS) [6] or hot isostatic pressing (HIP).

In the present work, the microstructure of $\mathrm{Cu}-10 \mathrm{nD}$ and $\mathrm{Cu}-10 \mathrm{G}$ composites has been characterized by transmission electron microscopy (Fig.1). The friction coefficient of the materials has been determined with A CSEM nanotribometer with a commercial steel ball counter body has been used to determine the friction coefficient of the $\mathrm{Cu}-\mathrm{C}$ composites (0.3-0.4). Scanning electron microscopy and atomic force microscopy have been employed to characterize the wear tracks resulting from the previous measurements (Fig. 2). The tracks on $\mathrm{Cu}-10$ nd SPS and $\mathrm{Cu}-10 \mathrm{G}$ HIP samples evidenced essentially abrasive wear, while the tracks on the $\mathrm{Cu}$ $\mathrm{nD}$ extruded material showed a significant presence of delamination (Fig. 2).

This work has been performed under the Contract of Association between EURATOM and Instituto Superior Técnico. Financial support was also received from the Fundação para a Ciência Tecnologia (FCT) through PTDC/CTM/100163/2008 and PEST-OE/CTM-UI0084/2011 grants.

[1] Nickchi, T. et al., Mater Design, 32, 3548, 2011.

[2] Gubarevich, A.V. et al., Diam Relat Mater, 14, 1549, 2005.

[3] Suryanarayana, C. et al., Progress in Materials Science, 46, 1, 2001.

[4] Niwase, K. et al., JIM, 36, 282, 1995.

[5] Nunes, D. et al., Mater Sci Eng A, 528, 8610, 2011.

[6] Nunes, D. et al., Mater Sci Forum, 636, 682, 2010. 

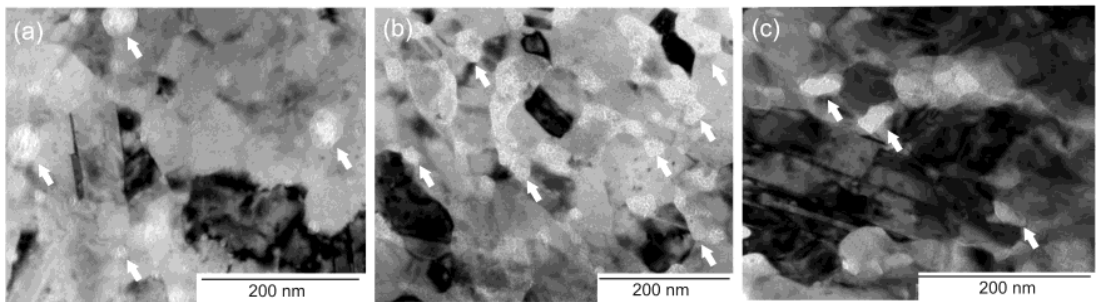

Figure 1. Transmission electron microscopy (bright-field) images of (a) $\mathrm{Cu}-10 \mathrm{nD}$ composite consolidated by SPS, (b) Cu-nD composite consolidated by hot extrusion $\left(600^{\circ} \mathrm{C}\right)$ and (c) $\mathrm{Cu}-10 \mathrm{G}$ consolidated by hot isostatic pressing (HIP). The arrows point to the carbon particles.
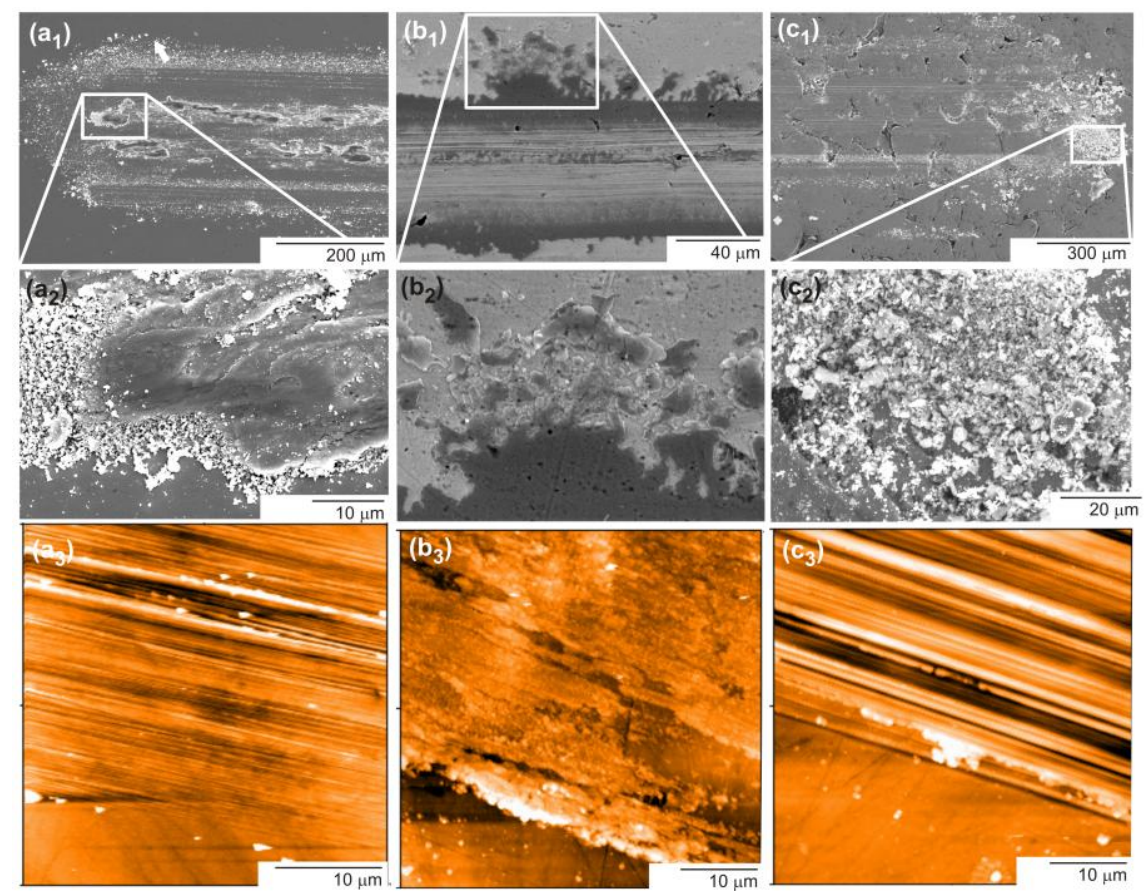

Figure 2. (a) $\mathrm{Cu}-10 \mathrm{nD}$ composite consolidated by SPS, (b) Cu-nD composite consolidated by hot extrusion and (c) $\mathrm{Cu}-10 \mathrm{G}$ consolidated by hot isostatic pressing (HIP). (1) Scanning electron microscopy (secondary electrons), (2) magnified detail, (3) atomic force microscopy (topography). 